\title{
EEG-based Decoding of Error-Related Brain Activity in a Real-World Driving Task
}

\author{
H. Zhang ${ }^{1}$, R. Chavarriaga ${ }^{1}$, Z. Khaliliardali ${ }^{1}$, L. Gheorghe ${ }^{1,2}$, I. \\ Iturrate $^{1}$ and J. d. R. Millán ${ }^{1}$ \\ ${ }^{1}$ Defitech Chair in Brain-Machine Interface, Center for Neuroprosthetics, School of \\ Engineering, École Polytechnique Fédérale de Lausanne (EPFL), Campus Biotech \\ H4, Chemin des Mines 9, Geneva CH-1202, Switzerland \\ 2 Nissan Motor Co., Ltd. Research Division, Research Planning Department, Atsugi, \\ Japan \\ E-mail: jose.millan@epfl.ch \\ October 2015
}

\begin{abstract}
.
Objectives. Recent studies have started to explore the implementation of braincomputer interfaces (BCI) as part of driving assistant systems. The current study presents an EEG-based BCI that decodes error-related brain activity. Such information can be used, e.g., to predict driver's intended turning direction before reaching road intersections.

Approach. We executed experiments in a car simulator $(N=22)$ and a real car $(N=8)$. While subject was driving, a directional cue was shown before reaching an intersection, and we classified the presence or not of an error-related potentials from EEG to infer whether the cued direction coincided with the subject's intention. In this protocol, the directional cue can correspond to an estimation of the driving direction provided by a driving assistance system. We analyze ERPs elicited during normal driving and evaluated the classification performance in both offline and online tests.

Results. An average classification accuracy of $0.698 \pm 0.065$ was obtained in offline experiments in the car simulator, while tests in the real car yielded a performance of $0.682 \pm 0.059$. The results were significantly higher than chance level for all cases. Online experiments led to equivalent performances in both simulated and real car driving experiments. These results support the feasibility of decoding these signals to help estimating whether the driver's intention coincides with the advice provided by the driving assistant in a real car.

Significance. The study demonstrates a BCI system in real-world driving, extending the work from previous simulated studies. As far as we know, this is the first online study in real car decoding driver's error-related brain activity. Given the encouraging results, the paradigm could be further improved by use of more sophisticated machine learning approaches and possibly be combined with applications in intelligent vehicles.
\end{abstract}

Keywords: brain-computer interface, error-related potentials, EEG, driving tasks, online decoding 


\section{Introduction}

Driving a vehicle requires multiple cognitive processes, e.g., sustaining current vehicle conditions, monitoring environmental events, and action decisions. Currently, driving assistant systems are mainly based on monitoring the vehicle conditions, e.g., the parameters of the car (steering, braking and accelerating), vehicle's location, complexity of the environment, and distance from other automobiles. Furthermore, it has been proposed that these systems can also monitor the driver's condition through the recording of physiological signals such as electroencephalography (EEG), electrocardiography (ECG) or electrooculography (EOG) (Chuang et al., 2010; Haufe et al., 2011; Haufe et al., 2014).

Brain-computer interfaces (BCI) have been developed to decode cerebral signals in order to restore communication capabilities for people with motor disabilities, or as an extra interaction mechanism for healthy individuals (Lebedev and Nicolelis, 2006; Millán and Carmena, 2010). One possible BCI application for able-bodied people is to integrate them into driving assistant systems. These BCI systems would decode driver's brain activity to estimate his/her cognitive states or action intentions. For instance, the system can verify whether the driver is paying attention to the driving behavior (Simon et al., 2011), estimate mental workload (Dijksterhuis et al., 2013), or predict driver's intention of action (e.g., braking, traffic lights, and lane changes) (Haufe et al., 2011; Gheorghe et al., 2013; Haufe et al., 2014; Sonnleitner et al., 2014; Kim et al., 2015; Khaliliardali et al., 2015).

The present study investigates another signal that may be used by driving assistance systems, i.e., the error-related brain activity. Error-related processing is a basic brain function related to learning and regulating goal-directed behavior (Holroyd and Coles, 2002; Taylor et al., 2007). It is considered as an underlying monitoring process for both endogenous and exogenous conflicting information, e.g., expectation mismatch or erroneous motor commission (Cavanagh et al., 2012). The error-related brain activity can be observed in scalp EEG recordings in the form of phase locked event-related potentials (ERP), around $80 \mathrm{~ms}$ after erroneous motor responses (Gehring et al., 1993; Holroyd and Coles, 2002; Cavanagh et al., 2012), or $250 \mathrm{~ms}$ after the stimulus onset in the case of monitoring of external errors (van Schie et al., 2004; Chavarriaga and Millán, 2010; Chavarriaga et al., 2014). This ERP pattern has been used in BCI for detecting error activity while human subjects either control moving objects (Parra et al., 2003; Ferrez and Millán, 2008) or monitor an external system (Chavarriaga and Millán, 2010; Iturrate et al., 2015). This information can then be used to correct user's erroneous decision (Parra et al., 2003), improve the information transfer rate of BCI system (Ferrez and Millán, 2008), or detect subject's intentional preferred target (Chavarriaga and Millán, 2010; Zhang et al., 2015; Iturrate et al., 2015). See Chavarriaga et al. (2014) for a review.

The current paper extends our previous study of detecting error-related brain activity in a car simulator (Zhang et al., 2013). In the experiments, a driving assistant 
system presents a directional cue indicating a turning direction before reaching an intersection, and single trial classification is performed to recognize error-related brain activity to verify whether the directional cue corresponds to the driver's intention (Zhang et al., 2013). In this study, we extend our previous work by performing a closed-loop experiment with online decoding of the error-related signals in the same setup. More importantly, in order to assess the feasibility of this protocol in a realistic driving scenario, we performed similar offline and online experiments in a real car with 8 participants.

\section{Materials and Methods}

\subsection{Participants}

Twenty-two subjects (three female, age $26.79 \pm 3.51$ ) participated in the experiments in the car simulator. Seven of them (one female, age $27.86 \pm 1.95$ ) participated in the evaluation of online decoding. Seventeen subjects in the experiment in the car simulator had driving licenses and nine of them were driving frequently.

Eight subjects (two female, age $28.62 \pm 2.72$ ) participated in the real car experiments. Seven of them (two female, age $28.42 \pm 2.88$ ) participated in the online evaluation. All subjects in the real car experiment held valid driving licenses and were active drivers. One subject participated in both the driving simulator and real car experiments. The experimental protocols were approved by the local ethical committee (EPFL-Brain and Mind Institute ethical committee) and subjects provided written informed consent. All subjects had normal or corrected-to-normal vision, and did not report any known neurological or psychiatric diseases.

\subsection{Experimental settings in car simulator}

The first set of experiments was performed on a custom-made simulated car, shown in Figure 1.A, corresponding to a realistic vehicle with normal-sized car seat, steering wheel and accelerate and brake pedals. For the experiments, we designed a 3D virtual environment of a small town including six north-to-south and five east-to-west roads arranged in a rectangular grid using the software Blender (http://www.blender.org/). The protocol was run in a customized open source driving simulation program (VDrift http://vdrift.net/). The virtual driving environment was presented in three 27 inch 3D monitors, which were about $50 \mathrm{~cm}$ away from subject's eyes. The monitors allowed to present the scene from 8 directions without requiring the use of 3D glasses. During the experiment, the car control signals (mainly steering and pedal positions), vehicle dynamics and its position in the virtual environment were continuously recorded at a sampling rate of $256 \mathrm{~Hz}$.

Subjects were instructed to drive following static street boards located on top of the intersections as shown in Figure 1.B. The speed of the vehicle was limited to $60 \mathrm{Km} / \mathrm{h}$. When the car was less than $80 \mathrm{~m}$ away from the intersection, a visual cue composed 
of three gray arrows (pointing left, up and right) appeared at the lower part of the windshield. One second later, and always before the car reached the intersection, one of the arrows was highlighted in green, indicating one of the possible driving directions (directional cue). This cue indicated the turning direction that may have been inferred by a driving assistance system. All cues disappeared $500 \mathrm{~ms}$ after the directional cue.

During the experiment, the probability of the directional cue (green arrow) pointing to the same direction as the street board was 70\%. Twenty-two subjects performed one offline recording composed of 5 runs, where each run was composed of 30 trials, i.e., 30 intersections, and lasted about 9 to 12 minutes. Seven of these subjects participated in a subsequent online recording in another day, which contained 4 to 6 runs each depending on the subject. This yielded to 150 trials for offline runs and an average of $158.57 \pm 14.64$ trials for the online analysis.

\subsection{Experimental settings in the real car}

The real car experiments were performed in an vehicle Infiniti FX30, shown in Figure 1.D. Subjects were requested to use the automatic gearshift and keep their hands on the steering wheel to limit their arm movements. We disabled the built-in driving assistance systems during the experiments, i.e., intelligent cruise control, lane departure prevention, and vehicle dynamic control. Similarly to the car simulator, the vehicle was equipped with a driving logger (provided by the manufacturer) that recorded steering and pedal positions at a sampling rate of $256 \mathrm{~Hz}$. A 7 -inch screen was placed on the dashboard in front of the subject (behind the steering wheel) to show the task-related arrows during the experiments. For safety reasons, the real driving experiments were performed in a closed road without any other vehicle or pedestrians.

Subjects drove the car following direction signs placed along the road, as shown in Figure 1.C and E. Based on the topology of the real road used for the experiments, the options of turning were reduced to left and right, thus only these two arrows were shown as directional cues. The timings of the visual cues were exactly the same as in the car simulator. Subjects were asked to drive normally as in a secondary road and limit their speed to $60 \mathrm{~km} / \mathrm{h}$. Each run of the real car recording consisted of 5 laps (9 trials per lap), i.e., 45 trials per run, which lasted around 15 to 20 minutes, depending on the speed of the car. We recorded 3 runs for each subject corresponding to 135 trials. Eight subjects took part in a first day of recording consisting of offline experiments, and seven of them participated in a second recording day to test the online decoding. Six subjects performed directly the online runs using a classifier trained in offline data from the previous day. The offline data (first day) of the remaining subject was contaminated by artifacts, therefore she performed two offline runs on the second day, followed by one online run.

In most runs of the online experiment with the real car (normally from the second run), subjects were given additional visual feedback $1 \mathrm{~s}$ after showing the directional cue (green arrow). The visual feedback was a red arrow, only shown when an error potential 

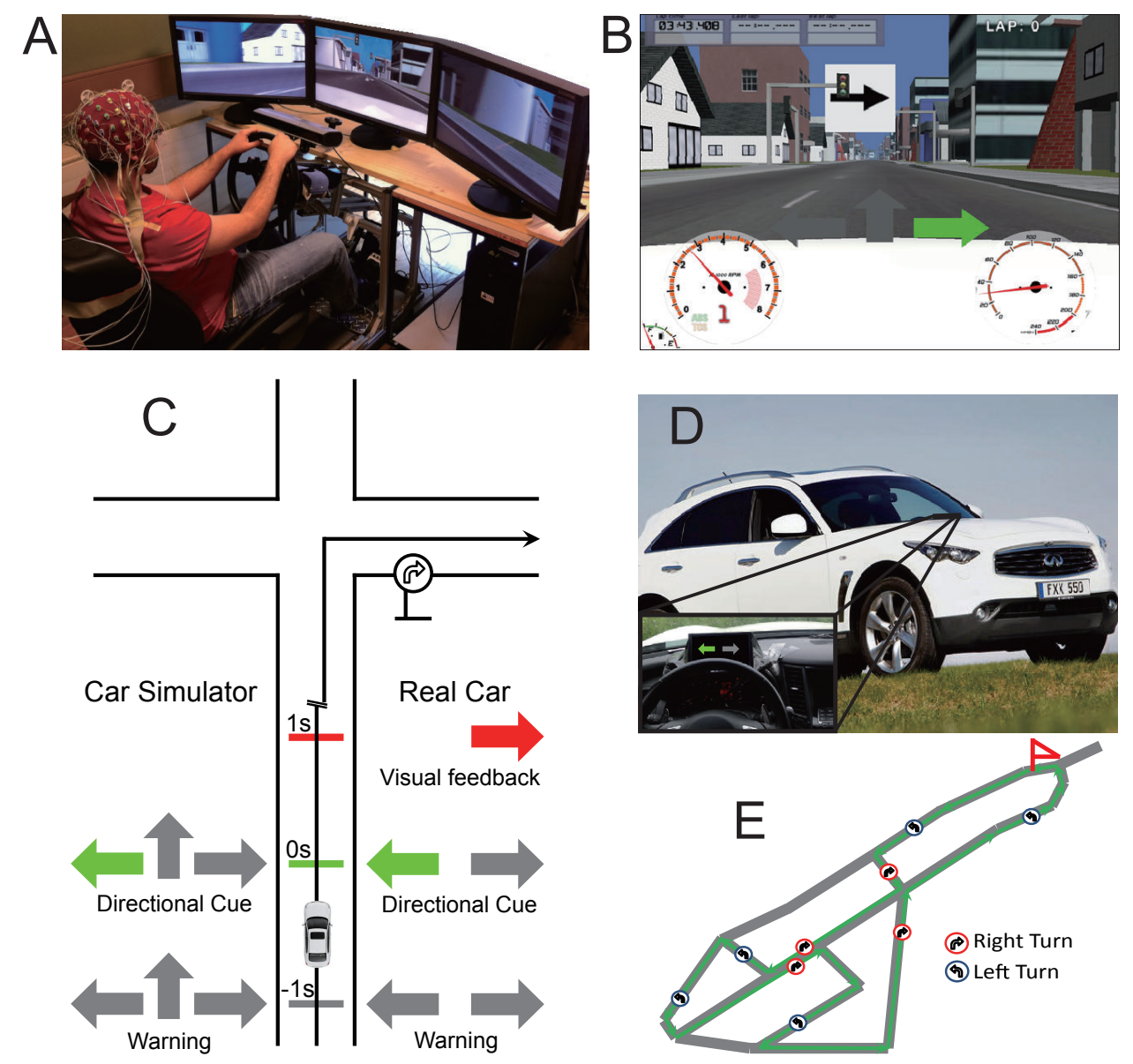

Figure 1. Experimental settings and protocols. A. Setup of the experiments in the car simulator together with the EEG recording system. B. Virtual environment as perceived by the driver in the car simulator. Directional signs (white panel with black arrow in the center) shows the direction of turning; while information from the driving assistant are shown over the dashboard (gray and green arrows). C. Timings for both experimental protocols. A visual cue is shown to notify subjects that a new trial is starting, and the directional cue informs a possible turning direction. Error-related brain activity is elicited if the arrows do not coincide with subject's preference. During the real car experiments, the visual feedback indicates whether the system has detected an error potential. D. Vehicle Infiniti FX30 used for the real car experiments. The inset shows the monitor displaying the experimental cues. E. The closed track used for the real car experiments. The red flag indicates the start point of the lap.

was detected from the EEG (Figure 1.C), pointing to the opposite direction as the green arrow. No visual feedback was shown when the EEG signal was recognized as a correct trial. The term "visual feedback" is used to indicate the red arrow after classification.

\subsection{Data acquisition and pre-processing}

Unless specified, the processing of physiological signals was the same for both simulated and real driving experiments. EEG signals were recorded from 64 locations according 
to the extended 10/20 system using a Biosemi Active Two system with a sampling rate of $2048 \mathrm{~Hz}$. Ground was replaced by the Driven Right Leg (DRL) passive electrode. Signals were referenced to the CMS electrode placed $1 \mathrm{~cm}$ to the left of POz. EOG signals were simultaneously recorded using electrodes positioned above the nasion and below the outer canthi of both eyes. Experimental events (timing and type of the visual cues) were marked in both EEG data and the driving log file via hardware triggers sent from a parallel port.

For the data obtained in the car simulator, we analyzed the temporal waveform of the ERP after filtering the raw signal spatially, using common average reference (CAR), and in the frequency domain, with a $4_{t h}$ order non-causal Butterworth filter with cutoff [1 10$] \mathrm{Hz}$. The choice of this frequency band is based on the fact that the main oscillatory signature of error-related brain activity appears in the theta band (Holroyd and Coles, 2002; Taylor et al., 2007; Chavarriaga et al., 2014). We kept the same processing for the online experiment in the car simulator, except for the use of a causal spectral filter. In addition to the analysis of the temporal waveform, we also computed the power spectrum density (PSD) of the signal in a broader band, namely [1 50] Hz for car simulator and [1 30] Hz for the real car. This difference was motivated by the presence of vehicle specific electrical noise at $30 \mathrm{~Hz}$. The PSD was computed by short-time Fourier transform in a sliding window. The window length was $500 \mathrm{~ms}$ (128 samples, sampling rate $=256 \mathrm{~Hz}$ ), and the moving step was $31.2 \mathrm{~ms}$ (4 samples). The computation was performed using Matlab function spectrogram with 256 points Fast Fourier Transform. The resulted PSD at time $\mathrm{T}$ was computed using the time window $\left(\begin{array}{ccc}T-250 & T+250\end{array}\right] \mathrm{ms}$.

For the real car experiment, the offline analysis was the same as in the car simulator. Prior to applying CAR, the EEG data was visually inspected to discard noisy channels. The signal in those channels was replaced by the average of neighbors. Contaminated electrodes were mainly located in parietal regions, which are [FC5 PO4 P2] for subject 1, [FC5] for subject 4, [P1 Pz P2 P3 CP2 CP4 CP6 FC6 O2] for subject 5, and [P1 PO4] for subject 7 .

For the online processing of the real car data, the CAR was not performed for the reason that the signals were more prone to contamination in some of the electrodes, and the contaminated signal could have affected other channels. Among all subjects, one of them (Subject 4) had a substantial noise level in the real car experiments on the first day. For this reason, we discarded the subject's data on that day and only used the data from the second day.

After preprocessing, we extracted correct and error trials according to the onset of the directional cue $(t=0 \mathrm{~s})$. Epochs were defined to include data from one second before $(\mathrm{t}=-1 \mathrm{~s})$ to one second after $(\mathrm{t}=1 \mathrm{~s})$.

\subsection{Classification}

EEG activity was decoded in order to infer whether the user perceived the directional cue as 'correct', i.e., in agreement with the intended direction showed by the traffic sign, 
or 'erroneous', otherwise. Classification between correct and error was performed based on the ERP time signal in the period, between $0.2 \mathrm{~s}$ and $0.7 \mathrm{~s}$ after the onset from 41 EEG electrodes: AF3, F1, F3, F5, FC5, FC3, FC1, C1, C3, C5, CP5, CP3, CP1, P1, P3, P5, PO3, POz, Pz, CPz, AF4, AFz, Fz, F2, F4, F6, FC6, FC4, FC2, FCz, Cz, C2, C4, C6, CP6, CP4, CP2, P2, P4, P6 and PO4 ${ }^{1}$. The 50 most discriminant features (channel and time point) were selected for classification. The discriminant power of each feature

was estimated using the Fisher score: $\frac{\left|m_{1}-m_{2}\right|}{\left(s_{1}+s_{2}\right)}$, where $m_{i}$ and $s_{i}$ are the mean value and variance of the samples from the $i^{\text {th }}$ class.

We used Linear Discriminant Analysis (LDA) to classify correct and error trials. The offline performance was evaluated using 10-fold cross validation, where the folds were generated keeping the temporal structure of the data. Feature selection was performed separately per each fold using the training data. Since the trial numbers for the two classes were not balanced, i.e., about $30 \%$ of them were error trials, we report the results in terms of both accuracy, and sensitivity-specificity in the receiver operating characteristics (ROC) space.

Furthermore, the results were compared with chance level, which was computed empirically by shuffling the training labels, and using the classifier modeled by such training data. The chance level is estimated from the performance distribution obtained by repeating the shuffling process 1000 times. We also controlled that the discriminative information was actually related to error processing in the brain and not caused by signal contamination due to eye movements. For this, we trained a classifier using the 3 EOG channels in the offline dataset for both car simulator and real car. The EOG channels were preprocessed in the same way as the EEG signal.

The online classification followed the same processing as the offline data. In the case of the car simulator, the classifier was trained using the data obtained so far on that day, i.e., the classifiers were trained on the data of the preceding runs: Data from the first run was used to train the initial classifier; Online classification in the $i^{\text {th }}$ run $(i>1)$ was performed with using a classifier trained on all recorded data so far, i.e., runs 1 to $i$-1. In the real car experiments, with the exception of Subject 4 , we trained the classifier using the data recorded on the first day. The fact of training and testing the classifier on different days allowed us to assess the consistence of the error-related patterns across time.

\section{Results}

\subsection{Event-related potentials}

Frontal central areas are considered as the main brain source for error-related brain activity (Holroyd and Coles, 2002; Taylor et al., 2007). We observed clear error-related modulations over these areas in the offline experiments, as illustrated in the grand average ERP across all subjects (offline recordings: $\mathrm{N}=22$ for the car simulator and $\mathrm{N}$

\footnotetext{
${ }^{1}$ Peripheral electrodes were excluded to reduce signal contamination.
} 
$=8$ for the real car experiments) at $\mathrm{FCz}$ in Figure 2. The ERP from the car simulator (Figure 2.A) exhibits similar amplitude as the real car data (Figure 2.B). The signal is smoother in the car simulator, which can be due to the higher number of participants and trials and the lower noise level in the experimental environment.

The ERP elicited in both experiments showed a positive peak after the visual cue, peaking at about $760 \mathrm{~ms}$ before the directional cue in the car simulator data $(670 \mathrm{~ms}$ in the real car condition). No statistically significant difference was found ( $\mathrm{p}>0.05$, twosample $t$-tests, corrected by a 1000 random permutation test) between error and correct trials in the period before the directional cue. After the appearance of the directional cue $(0 \mathrm{~ms})$, another positive peak is found for both the car simulator and real car data, appearing around $260 \mathrm{~ms}$ and $200 \mathrm{~ms}$ for each experiment. Again, this peak does not show statistical difference between correct and error conditions, suggesting that none of these peaks is related to brain error processing. Specifically for the first peak (at $-760 \mathrm{~ms}$ and $-670 \mathrm{~ms}$ in the simulated and real car experiments, respectively), the directional cue (containing the information required to assess correct or erroneous conditions) has not yet been developed. In the experiments, the trials were randomly generated and balanced, i.e., left, right and straight arrows (left and right in the real car case) occur equally often in both error and correct conditions, so we could infer that these two peaks are not correlated with the perception of spatial patterns of the stimuli.

A positive peak at $290 \mathrm{~ms}$ in the real car data was also found, which is significantly different $(\mathrm{p}<0.05)$ between the error and correct trials. We observed a negative peak at about $480 \mathrm{~ms}$, having significantly lower amplitude in the error than the correct condition $(\mathrm{p}<0.05)$ for both the car simulator and the real car experiments. Significant differences between error and correct could also be found at around $700 \mathrm{~ms}$ after the directional cue with higher amplitude in the error condition. In summary, both experiments showed consistent ERP patterns and significant differences between the two conditions.

The topography of the 41 electrodes averaged for all subjects is presented in Figure 3. They show the scalp wide activity at the onset of the directional cue $(0 \mathrm{~s})$ and at the time of the ERP peaks. Frontal regions show a positive modulation after the visual cue (at $-760 \mathrm{~ms}$ in the car simulator and $-670 \mathrm{~ms}$ in the real car case) and the directional cue (260 ms for car simulator and $290 \mathrm{~ms}$ for the real car). High activity is observed after the visual cue, probably due to the fact that the subjects perceived suddenly the first visual cue (three gray arrows), which was also the purpose of presenting this visual cue, informing the subjects about the forthcoming directional cue in order to reduce the effect of visual surprise from the brain error processing. Error and correct trials showed similar patterns at $-760 \mathrm{~ms}$, confirming that no error-related information is contained. Topographies at the onset of the directional cue $(0 \mathrm{~ms})$ showed no specific modulation patterns, amplitudes being close to zero for all electrodes. We observed frontal negative deflections at about $\mathrm{t}=480 \mathrm{~ms}(\mathrm{t}=475 \mathrm{~ms}$ for the real car case) for error and correct conditions, with error trials having lower amplitude. Further, at about $\mathrm{t}=750 \mathrm{~ms}(\mathrm{t}=$ $700 \mathrm{~ms}$ for the real car case) we could see a positive pattern in frontal regions, especially in the error condition, corresponding to the positive peak in $\mathrm{FCz}$ in Figure 2. 

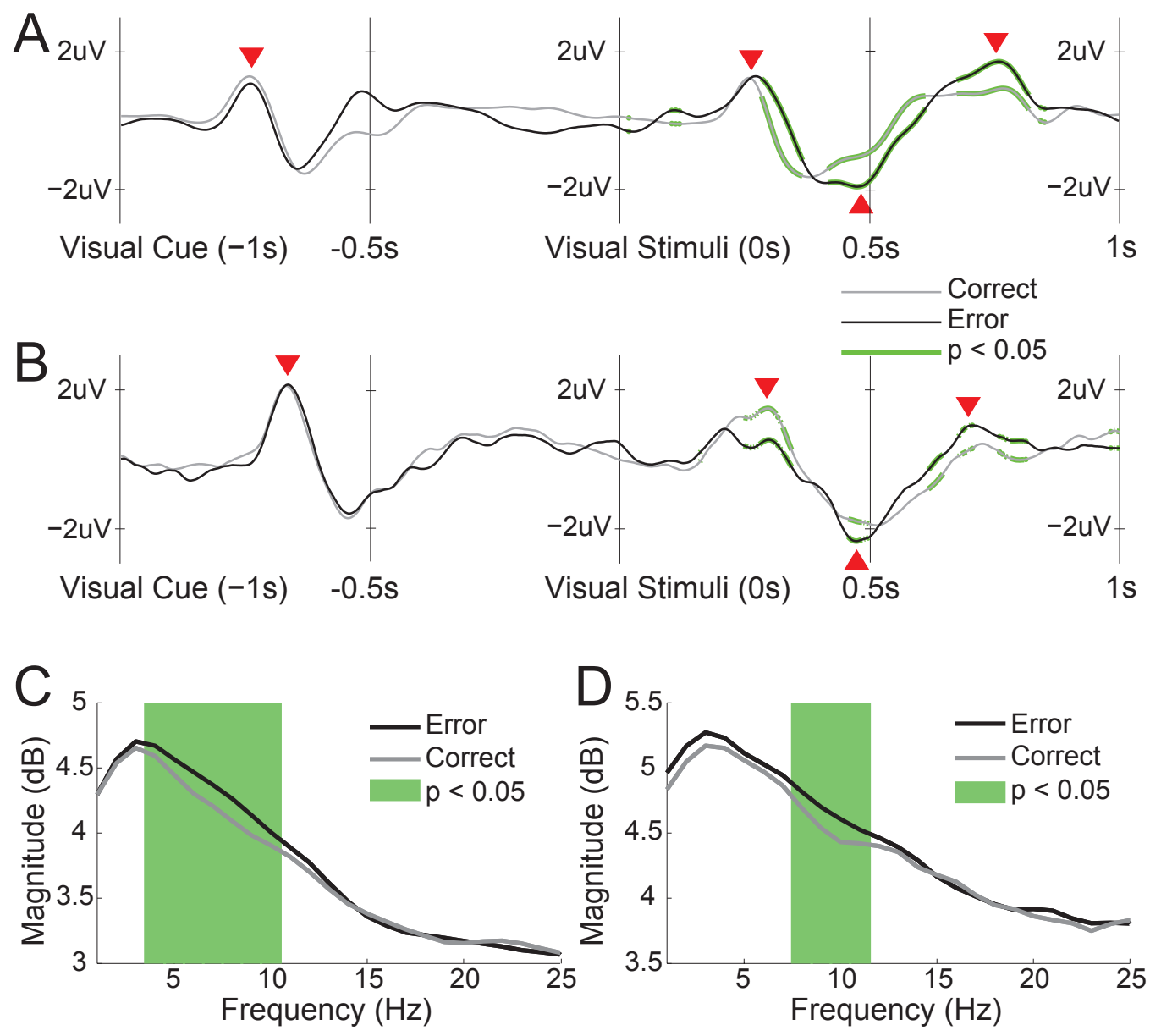

Figure 2. Grand average of ERP and PSD at FCz. EEG trials are epoched according to the directional cue $(0 \mathrm{~s})$, from $-1 \mathrm{~s}$ (presence of the three gray arrows) to $1 \mathrm{~s}$. A. ERP of the car simulator data. B. ERP of the real car data. Significant differences (p $<0.05)$ across time samples between error and correct trials are found by two-sample $t$-test for all trials, which are shown as green thick lines. The red triangles indicate the time points selected for the illustration of topography in Figure 3. C. PSD of the car simulator data. D. PSD of the real car data. PSD is computed in time window [200 400] ms. Statistical tests are performed by a two-sample $t$-test for all trials, and the significances $(\mathrm{p}<0.05)$ are shown as the green area.

The increase of the theta band in medial frontal cortex (MFC) is considered as the main oscillatory modulation pattern of error monitoring (Holroyd and Coles, 2002; Taylor et al., 2007; Cavanagh and Frank, 2014). Figure 2.C and D illustrate the power spectrum density (PSD) in the period [200 400] ms after the directional cue for electrode FCz. For both error and correct trials, the PSD curves decrease gradually, and drop after $12 \mathrm{~Hz}$. Both experiments showed higher theta power in the error condition. Specifically, significant differences $(\mathrm{p}<0.05$, two-sample $t$-test for all trials, corrected by a 1000 random permutation test) are found between $4-11 \mathrm{~Hz}$ (Figure 2.C) in the car simulator and between $7-11 \mathrm{~Hz}$ in the real car (Figure 2.D) case. No difference could be found in higher frequency ranges. 


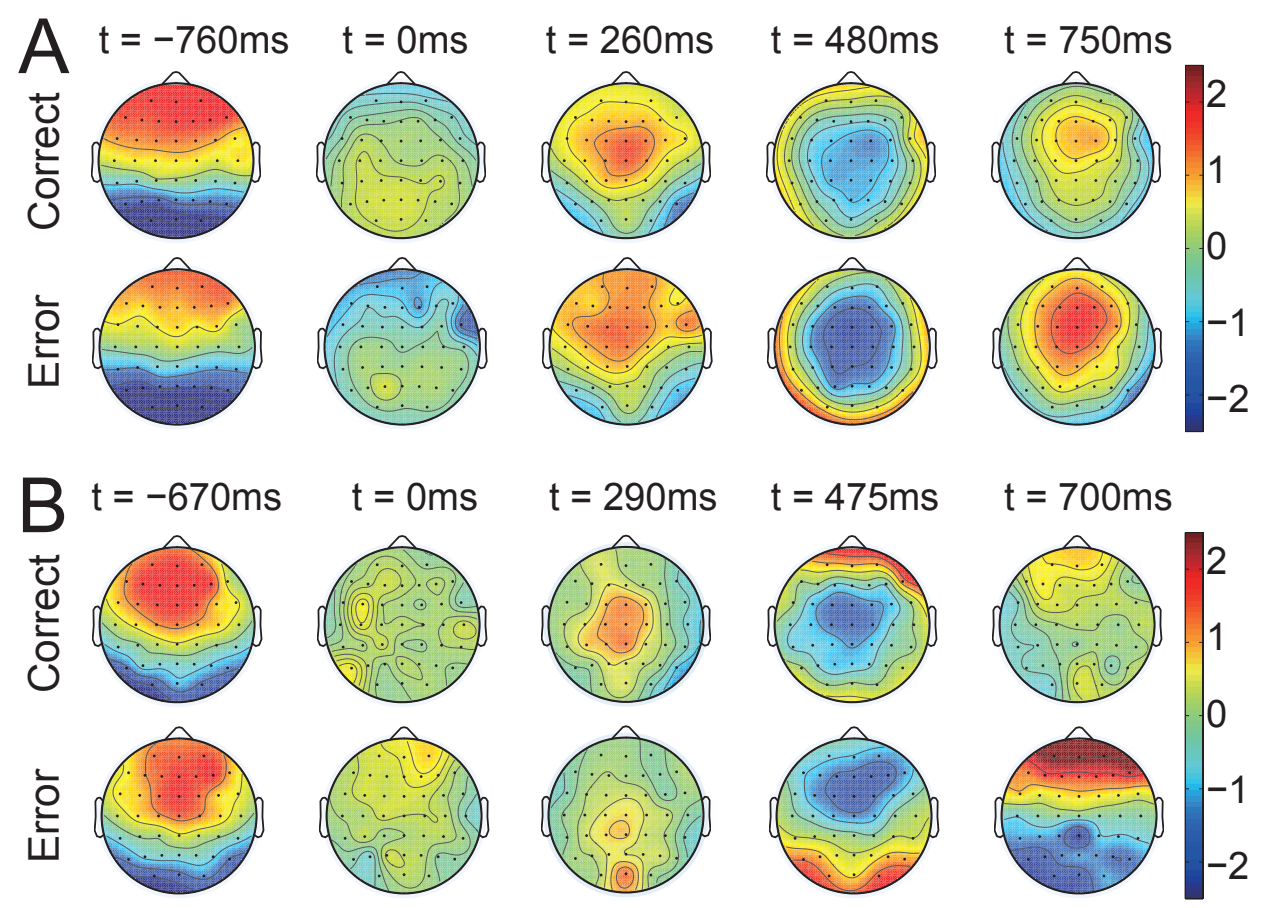

Figure 3. Topographic illustration of brain activity at time points of ERP peaks and $\mathrm{t}=0 \mathrm{~ms}$ (onset of the directional cue). A. Car simulator dataset. B. Real car dataset. 41 central electrodes are shown in the figure, excluding the peripheral regions.

\subsection{Offline classification results}

The results of offline classification are shown in Figure 4. Each curve in Figure 4.A and $\mathrm{B}$ represents the performance of one subject in the ROC space, averaged across 10 folds. In both experiments most of the subjects had a classification performance above 0.5 (diagonal dash line). The average accuracy across subjects was $0.698 \pm 0.065$ and $0.6823 \pm 0.059$ for the car simulator $(\mathrm{N}=22)$ and real car $(\mathrm{N}=8)$ experiments, respectively. The sensitivity-specificity of the decoder using the area under the ROC curve (AUC) was of $0.729 \pm 0.086$ and $0.6824 \pm 0.086$ for the car simulator and real car experiments. Although the accuracy and AUC were lower in the real car, no significant difference (two sample $t$-test, $\mathrm{p}>0.05$ ) was found between the two experimental settings.

Furthermore, we computed the chance level for both experimental settings, see white boxplot in Figure 4.C. It yielded a chance accuracy of $0.523 \pm 0.042$ (car simulator) and $0.554 \pm 0.030$ (real car), and AUC of $0.494 \pm 0.050$ (car simulator) and $0.526 \pm$ 0.051 (real car). Significant differences ( $\mathrm{p}<10^{-4}$, paired $t$-test) were found between the chance level (blank boxes) and the EEG classification for both accuracies and AUC. The performance with EOG features is illustrated as gray boxplots in Figure 4.C and $\mathrm{D}$, which were significantly lower (one sample $t$-test, $\mathrm{p}<0.05$ ) than using EEG features for both accuracy $(0.614 \pm 0.082$ for the car simulator and $0.589 \pm 0.106$ for the real car) and AUC (0.616 \pm 0.116 for the car simulator and $0.574 \pm 0.142$ for the real car).

To further rule out any influence of EOG on performance, we compared 
classification using EEG features against using EEG + EOG features. Results were statistically similar for both, car simulator (AUC $0.723 \pm 0.092$; Accuracy $0.683 \pm$ 0.082 ) and real car experiments (AUC $0.680 \pm 0.093$; Accuracy $0.665 \pm 0.081$ ). We also explored the use of ICA for removing EOG before classifying the denoised EEG. To do so, we removed the ICA components correlated with veritcal and horizontal eye movements (correlation coefficient $>0.2$ ). The horizontal and vertical movements were estimated as $\left[s_{2}-s_{3}\right]$ and $\left[s_{1}-\left(s_{2}+s_{3}\right) / 2\right]$, where $s_{1}, s_{2}$ and $s_{3}$ are the signals from the three EOG channels located above nasion $\left(s_{1}\right)$ and below the out canthi of both eyes $\left(s_{2}\right.$ and $\left.s_{3}\right)$. Classification using ICA-denoised brain signals yielded an AUC of 0.698 \pm 0.129 and $0.689 \pm 0.072$ (Accuracy: $0.670 \pm 0.102$ and $0.663 \pm 0.060$ ) for the car simulator and the real car experiments, showing no significant performance differences with the decoding of the original EEG signals.

\subsection{Online classification}

For the experiments in the car simulator, since the classifier was updated after every run, we report online performance for each run, from runs 2 to run 5, c.f., black boxes in Figure 5.A. The later run has higher accuracy than the previous ones; thus, the $5^{\text {th }}$ run $(0.733 \pm 0.150)$ has higher accuracy than the $2^{\text {nd }}$ run $(0.567 \pm 0.101)$, which is statistically significant $(\mathrm{p}<0.05$, paired t-test). The white boxes in Figure 5 represent the chance levels in these runs (by randomly shuffling the training labels and evaluating the classification performance in each run, averaged for 1000 times), where the third and fifth runs have significantly higher performance compared with the chance level. The false positive rate and true positive rate of each run for the seven subjects are illustrated in Figure 5.B, showing that the performance exceeds chance level in most runs. We could also observe substantial differences between subjects, e.g., the subject corresponding to blue diamond shows better performance than that of green hexagrams, which might be due to the diversity of the signal quality, the experience of performing similar experiments and the attention level during the recording.

Similarly, the results of online real car experiments are shown in Figure 6. Since the online experiments have different number of runs across subjects, we present the results of each run for each participant separately. Five subjects did the first online run without the visual feedback, i.e., the red arrow one second after detecting the errorrelated brain activity. Excepting subject 4, the classifiers were trained based on the data collected in the previous recording day, and no further update of the classifier was done during online experiments. As shown in Figure 6.A, the classification performance increased across runs, particularly the first run never led to the best accuracy for any subject. Again, variations in performance existed across participants, e.g., subject 2 had better performance $(0.786 \pm 0.060)$ than the others, and subject $3(0.545 \pm 0.068)$ has performance close to random level except for the last run. As mentioned in the section 2.3 , subject 4 has only one online run using the training data of the same day. This might be one of the reasons why this subject has relatively higher performance (0.773) 

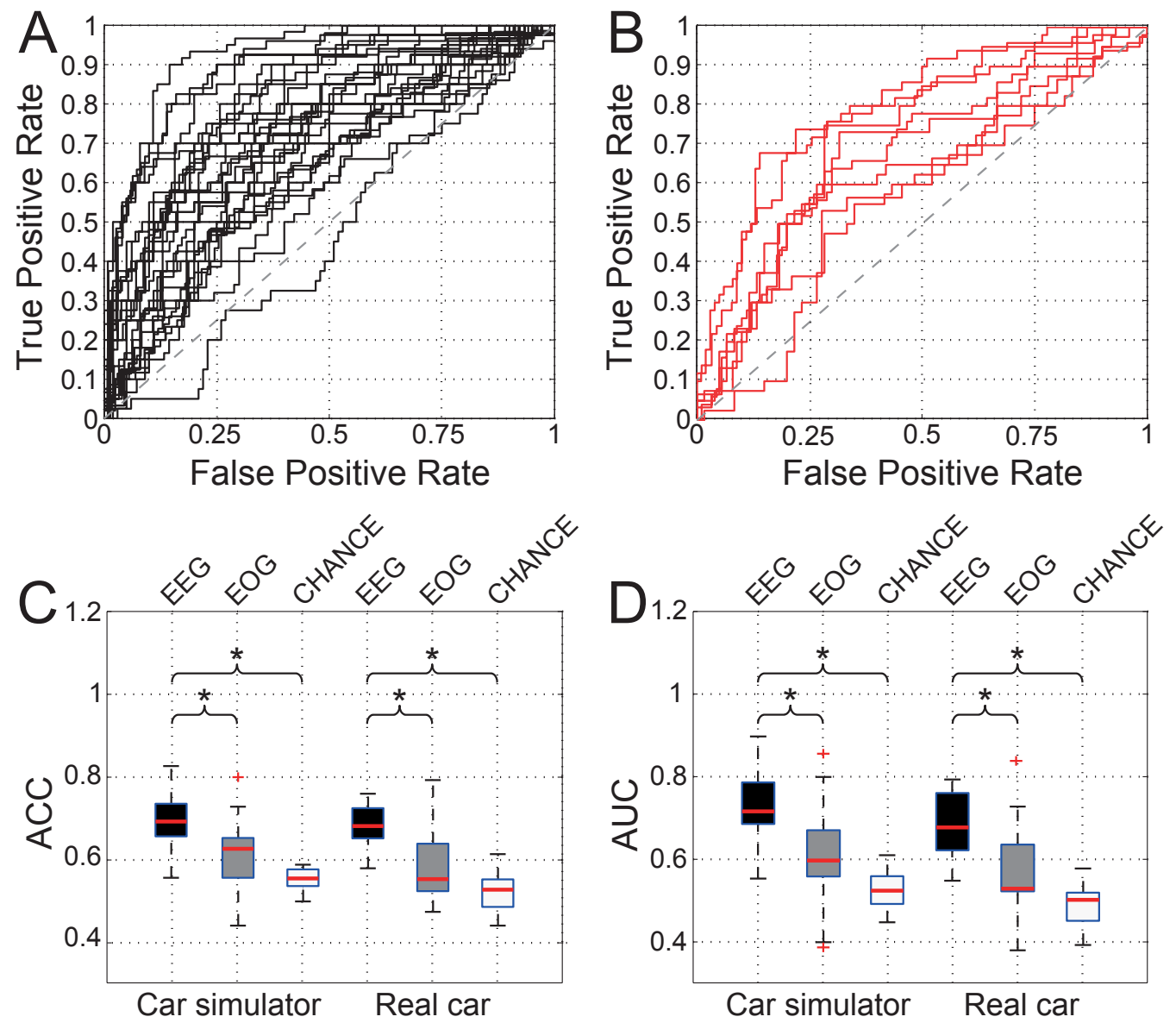

Figure 4. Offline classification performance of both car simulator $(\mathrm{N}=22)$ and real car data $(\mathrm{N}=8)$. A. Results in ROC space for the car simulator, i.e., quantified by false positive rate and true positive rate. Each curve shows results of one subject (10-fold cross validation) B. Results in ROC space for the real car. C and D. Median and 25 th/75th percentiles of accuracy and area under the curve in car simulator and real car datasets. Black boxes show the results of using EEG signal, gray boxes show the results of using EOG signal, and the empty boxes indicate the chance levels. Asterisk indicates significant difference using $t$-test.

than other subjects.

\section{Discussion}

In this study, we have analyzed and decoded error-related brain activity while driving a simulated and a real car. Classification of the EEG signals in a single trial basis yielded performances significantly above chance level for most subjects and therefore can be used to obtain information about the driver's appraisal of the presented stimuli, e.g., to validate inferences made by a driving assistant system. This study extends our previous work from car simulator to real car experiments. The consistency of ERP grand averages and classification performance between the car simulator and real car 
experiments supports the idea of using BCI systems to assist in driving tasks. More importantly, the current study also evaluated decoding of these signals in an online manner, showing similar performance between the two experimental setups, further proving the feasibility of such systems in realistic applications.

Unsurprisingly, the EEG signals were more prone to artifact contamination in the real car situation than in the simulated condition. The environmental noise, e.g., moving and shaking of the automobile as well as the movements of the subject's eyes and head are some of the factors that decrease the quality of the EEG data in this condition. Concerning these aspects, the robustness and reliability should be taken into account in the design of in-car BCI systems. The error-related EEG activity has been reported to be largely independent from the stimuli type (e.g., visual, auditory or tactile) and spatial position of stimuli, modulated mainly by the subjects' perception of conflicting information (Chavarriaga and Millán, 2010). This activity is mainly associated with modulations in theta band, thus the interest frequency range did not overlap with the vehicle specific electrical noise we observed $(30 \mathrm{~Hz}$ in the real car experiments). The fact that EOG-based classification yielded low performance also supports the notion that the discriminative patterns are not originated from eye movements. Nevertheless, further studies and future applications may attempt to apply algorithms to remove eye movements and blinks automatically (Joyce et al., 2004).

The classification results showed performance variations across subjects and runs. In general, most subjects were naive to the protocol, both for the car simulator and the real car experiments. Only one subject performed the experiment in the car
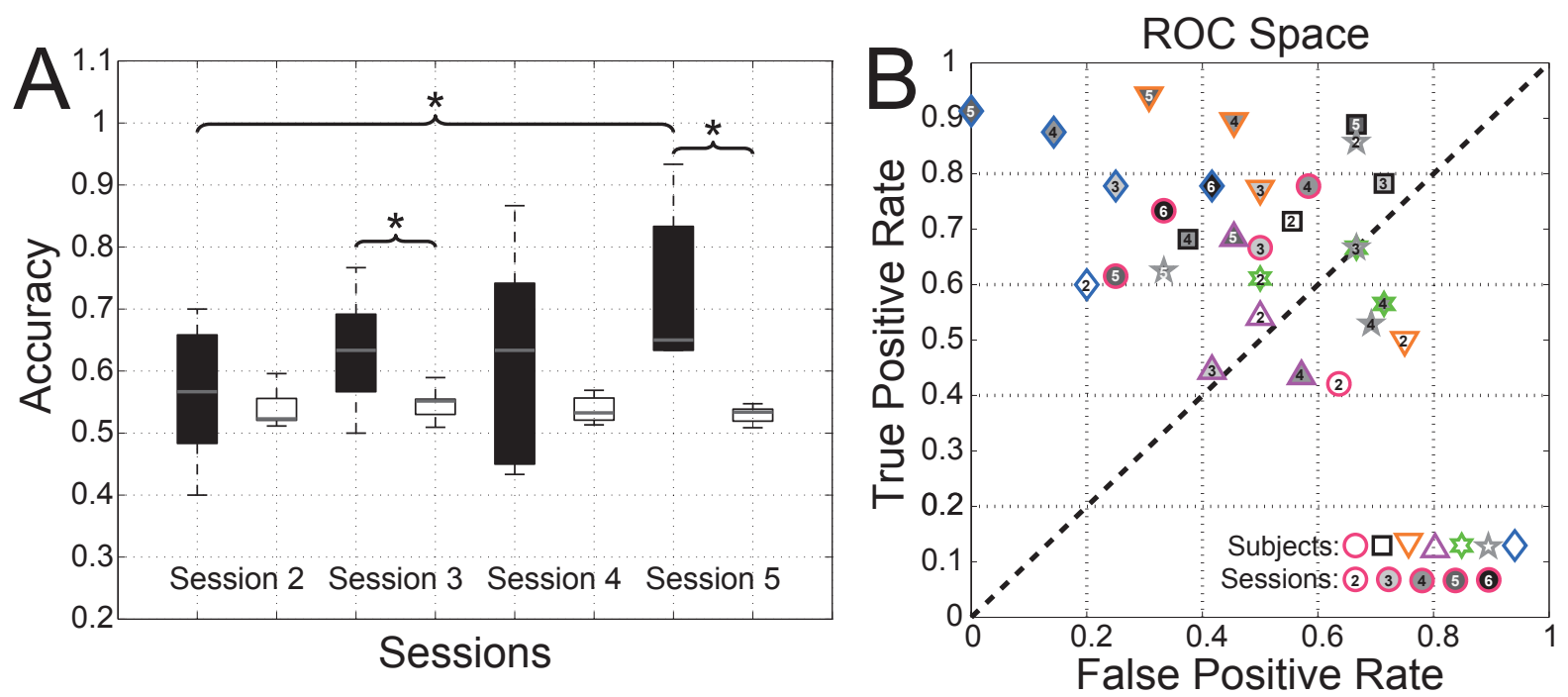

Figure 5. Performance of online experiments in the car simulator $(\mathrm{N}=7)$. A. Classification accuracies in the online runs (from 2 to 5), averaged across participants. Error bars represents the standard deviation. White boxes indicate the chance level. B. False positive and true positive rate of all online runs for each subject. Each type of marker represents one specific subject and the face color (white to black) indicates the run order, i.e., lighter markers correspond to earlier runs than darker markers. 

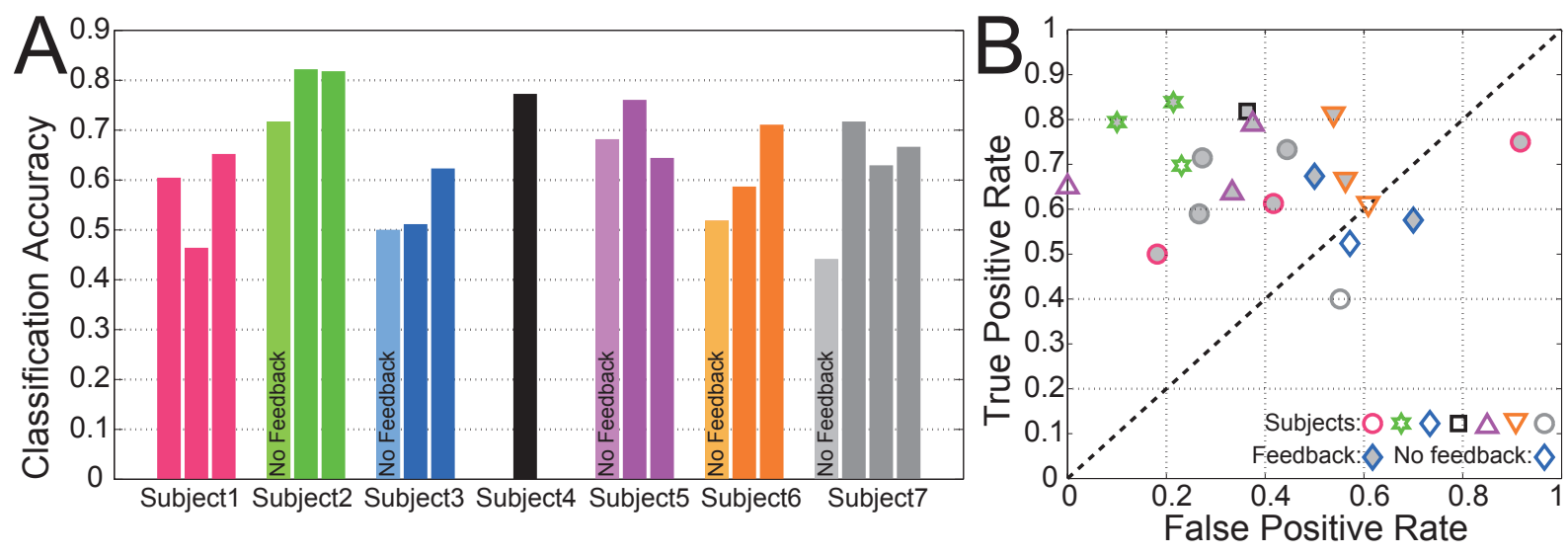

Figure 6. Online performance of real car experiments $(\mathrm{N}=7)$. A. Classification accuracy for each subject with different number of runs. Runs that did not deliver visual feedback was labeled as 'No Feedback'. B. False positive and true positive rate of all runs. Subjects are represented by marker types, and the color convention is the same as in A. The runs with feedback are indicated with filled markers.

simulator before the real car recording. The performance variability across individuals and runs may be caused by differences in the attention level and the adaptation to the protocol. As we saw in the car simulator data (Figure 5.A), the performance gradually and significantly increased from run 2 to run 5, which is also supported by the real car dataset where later runs outperformed the first one. These results suggest that a certain adaptation is necessary for the subject to obtain good and stable classification performance. Nevertheless, we cannot exclude that the increase in accuracy observed in the online car simulator experiments are not due to the larger amount of data available for training the decoder. Further studies may try to find the relative contribution of data amount and subject adaptation.

Comparing our results with a previous study on error monitoring in the laboratory environment (Chavarriaga and Millán, 2010), whose mean classification accuracies were 0.758 and 0.632 for correct and error trials, we observed no obvious drop of the classification performance. This proves, therefore, that detecting the error-related brain activity in complex tasks is feasible and the accuracy is equivalent to that of simpler setups.

To increase the classification performance in the future, we can improve the feature selection method in order to reduce redundancy across features (Brown et al., 2012). Additionally, the current study only uses the discriminative information from ERP patterns, or temporal waveforms, as classification features. Alternative features, e.g., spectral information and causal influences between electrodes, are likely to boost classification performance according to some reported studies on BCI (Wang et al., 2006; Billinger et al., 2013; Omedes et al., 2014; Zhang et al., 2015). Moreover, the online results in the car simulator data indicate that the prompt updating of the classifier seems to contribute to performance improvement, which might be further evaluated through the adaption of the classifier parameters, e.g., weights in an LDA classifier (Hsu, 2011). 
These sets of techniques could also help coping with changes in the EEG induced by long-term driving, which may affect the decoding performance. Still, although ERP amplitudes may decrease, their waveform appear to be rather stable despite mental fatigue (Lorist et al., 2005). This issue will be considered in future studies.

Finally, it should be noticed that the BCI system developed in this study is not intended for direct control of the vehicle (e.g., steer, accelerate and brake). Instead, the decoding of error-related brain activity shows whether the driver agrees with the assistance provided by the vehicle. Furthermore, such error-related activity could not only infer the driver's immediate response to the assistance, but can also be used to gradually adapt the driving assistance for future occasions (Iturrate et al., 2015).

In summary, we have presented the first online BCI system tested in a real car to detect error-related brain activity, as a first step in transferring error-related BCI technology from laboratory studies to the real-world driving tasks. Consistent brain signatures and classification performance prove the feasibility of the approach in complex environments, as an extension of the previous study in a car simulator. Our future work will focus on investigating other machine learning methods to improve the performance of error detection, evaluating the possibility of using alternative stimuli, e.g., auditory or tactile, to evoke error-related brain activity without extra visual burden during driving, as well as testing the protocol in open roads as compared to driving in a closed track.

\section{Acknowledgments}

The authors of this paper like to thank all participants. Real car experiments were possible thanks to the support of the EPFL transportation center (TraCE), and the Cantonal road service of Vaud, Switzerland. We acknowledge the valuable assistance and support from Michaël Themans (EPFL TraCE) and Jonathan Martin (Cantonal road service). This study was supported by Nissan Motor Co. Ltd., under the Research on Brain Machine Interface for Drivers project. H.Z. was partially supported by the Hasler foundation.

\section{References}

Billinger M, Brunner C, Müller-Putz G (2013) Single-trial connectivity estimation for classification of motor imagery data. J Neural Eng 10:046006.

Brown G, Pocock A, Zhao MJ, Luján M (2012) Conditional likelihood maximisation: a unifying framework for information theoretic feature selection. J Mach Learn Res 13:27-66.

Cavanagh JF, Frank MJ (2014) Frontal theta as a mechanism for cognitive control. Trends Cogn Sci 18:414-421.

Cavanagh JF, Zambrano-Vazquez L, Allen JJB (2012) Theta lingua franca: a common mid-frontal substrate for action monitoring processes. Psychophysiology 49:220-238.

Chavarriaga R, Millán JdR (2010) Learning from EEG error-related potentials in noninvasive braincomputer interfaces. IEEE Trans Neural Syst Rehabil Eng 18:381-388.

Chavarriaga R, Sobolewski A, Millán JdR (2014) Errare machinale est: the use of error-related potentials in brain-machine interfaces. Front Neurosci 8:208. 
Chuang CH, Lai PC, Ko LW, Kuo BC, Lin CT (2010) Driver's cognitive state classification toward brain computer interface via using a generalized and supervised technology In Proc Int Jt Conf Neural Netw.

Dijksterhuis C, de Waard D, Brookhuis KA, Mulder BLJM, de Jong R (2013) Classifying visuomotor workload in a driving simulator using subject specific spatial brain patterns. Front Neurosci 7:149.

Ferrez PW, Millán JdR (2008) Error-related EEG potentials generated during simulated braincomputer interaction. IEEE Trans Biomed Eng 55:923-929.

Gehring WJ, Goss B, Coles MGH, Meyer DE, Donchin EA (1993) Neural system for error-detection and compensation. Psychol Sci 4:385-390.

Gheorghe L, Chavarriaga R, Millán JdR (2013) Steering timing prediction in a driving simulator task. In Conf Proc IEEE Eng Med Biol Soc.

Haufe S, Treder MS, Gugler MF, Sagebaum M, Curio G, Blankertz B (2011) EEG potentials predict upcoming emergency brakings during simulated driving. J Neural Eng 8:1-11.

Haufe S, Kim JW, Kim IH, Sonnleitner A, Schrauf M, Curio G, Blankertz B (2014) Electrophysiology-based detection of emergency braking intention in real-world driving. J Neural Eng 11:056011.

Holroyd CB, Coles MGH (2002) The neural basis of human error processing: Reinforcement learning, dopamine, and the error-related negativity. Psychol Rev 109:679-709.

Hsu WY (2011) Eeg-based motor imagery classification using enhanced active segment selection and adaptive classifier. Comput Biol Med 41:633-639.

Iturrate I, Chavarriaga R, Montesano L, Minguez J, Millán JdR (2015) Teaching brain-machine interfaces as an alternative paradigm to neuroprosthetics control. Sci Rep 5:13893.

Joyce CA, Gorodnitsky IF, Kutas M (2004) Automatic removal of eye movement and blink artifacts from EEG data using blind component separation. Psychophysiology 41:313-325.

Khaliliardali Z, Chavarriaga R, Gheorghe LA, del R Millán J (2015) Action prediction based on anticipatory brain potentials during simulated driving. J Neural Eng 12:066006.

Kim IH, Kim JW, Haufe S, Lee SW (2015) Detection of braking intention in diverse situations during simulated driving based on EEG feature combination. J Neural Eng 12:016001.

Lebedev MA, Nicolelis MAL (2006) Brain-machine interfaces: Past, present and future. Trends Neurosci 29:536-546.

Lorist MM, Boksem MA, Ridderinkhof KR (2005) Impaired cognitive control and reduced cingulate activity during mental fatigue. Cogn Brain Res 24:199-205.

Millán JdR, Carmena J (2010) Invasive or noninvasive: Understanding brain-machine interface technology. IEEE Eng Med Biol Mag 29:16-22.

Omedes J, Iturrate I, Montesano L (2014) Brain connectivity in continuous error tasks In Conf Proc IEEE Eng Med Biol Soc.

Parra LC, Spence CD, Gerson AD, Sajda P (2003) Response error correction-a demonstration of improved human-machine performance using real-time EEG monitoring. IEEE Trans Neural Syst Rehabil Eng 11:173-177.

Simon M, Schmidt EA, Kincses WE, Fritzsche M, Bruns A, Aufmuth C, Bogdan M, Rosenstiel W, Schrauf M (2011) EEG alpha spindle measures as indicators of driver fatigue under real traffic conditions. Clin Neurophysiol 122:1168-1178.

Sonnleitner A, Treder MS, Simon M, Willmann S, Ewald A, Buchner A, Schrauf M (2014) EEG alpha spindles and prolonged brake reaction times during auditory distraction in an on-road driving study. Accid Anal Prev 62:110-118.

Taylor SF, Stern ER, Gehring WJ (2007) Neural systems for error monitoring: Recent findings and theoretical perspectives. Neuroscientist 13:160-172.

van Schie HT, Mars RB, Coles MGH, Bekkering H (2004) Modulation of activity in medial frontal and motor cortices during error observation. Nat Neurosci 7:549-554.

Wang Y, Hong B, Gao X, Gao S (2006) Phase synchrony measurement in motor cortex for classifying single-trial EEG during motor imagery. In Conf Proc IEEE Eng Med Biol Soc. 
Zhang H, Chavarriaga R, Gheorghe L, Millán JdR (2013) Inferring driver's turning direction through detection of error related brain activity. In Conf Proc IEEE Eng Med Biol Soc.

Zhang H, Chavarriaga R, Millán JdR (2015) Discriminant brain connectivity patterns of performance monitoring at average and single-trial levels. NeuroImage 120:64-74. 